\title{
Fibroblast growth factor 21 participates in adaptation to endoplasmic reticulum stress and attenuates obesity-induced hepatic metabolic stress
}

\author{
Seong Hun Kim • Kook Hwan Kim • Hyoung-Kyu Kim • \\ Mi-Jeong Kim • Sung Hoon Back • Morichika Konishi • \\ Nobuyuki Itoh • Myung-Shik Lee
}

Received: 30 July 2014 / Accepted: 25 November 2014 / Published online: 24 December 2014

(C) Springer-Verlag Berlin Heidelberg 2014

\begin{abstract}
Aims/hypothesis Fibroblast growth factor 21 (FGF21) is an endocrine hormone that exhibits anti-diabetic and anti-obesity activity. FGF21 expression is increased in patients with and mouse models of obesity or nonalcoholic fatty liver disease (NAFLD). However, the functional role and molecular mechanism of FGF21 induction in obesity or NAFLD are not clear. As endoplasmic reticulum (ER) stress is triggered in obesity and NAFLD, we investigated whether ER stress affects FGF21 expression or whether FGF21 induction acts as a mechanism of the unfolded protein response (UPR) adaptation to ER stress induced by chemical stressors or obesity.
\end{abstract}

Electronic supplementary material The online version of this article (doi:10.1007/s00125-014-3475-6) contains peer-reviewed but unedited supplementary material, which is available to authorised users.

S. H. Kim $•$ K. H. Kim $(\bowtie) \cdot$ M.-S. Lee $(\bowtie)$

Department of Medicine, Samsung Medical Center, Sungkyunkwan University School of Medicine, 81 Irwon-ro, Gangnam-gu,

Seoul 135-710, South Korea

e-mail:kookhwankim@gmail.com

e-mail: mslee0923@skku.edu

S. H. Kim $・$ K. H. Kim • M.-S. Lee

Samsung Advanced Institute for Health Sciences and Technology,

Sungkyunkwan University School of Medicine, Seoul, South Korea

H.-K. Kim • M.-J. Kim • S. H. Back

School of Biological Sciences, University of Ulsan,

Ulsan, South Korea

\section{Konishi}

Department of Microbial Chemistry, Kobe Pharmaceutical

University, Motoyamakita-machi, Higashinada-ku, Kobe, Japan

N. Itoh

Department of Genetic Biochemistry, Kyoto University Graduate

School of Pharmaceutical Sciences, Sakyo, Kyoto, Japan
Methods Hepatocytes or mouse embryonic fibroblasts deficient in UPR signalling pathways and liver-specific eIF2a mutant mice were employed to investigate the in vitro and in vivo effects of ER stress on FGF21 expression, respectively. The in vivo importance of FGF21 induction by ER stress and obesity was determined using inducible $F g f 21$-transgenic mice and Fgf21-null mice with or without leptin deficiency. Results We found that ER stressors induced FGF21 expression, which was dependent on a PKR-like ER kinaseeukaryotic translation factor $2 \alpha$-activating transcription factor 4 pathway both in vitro and in vivo. Fgf21-null mice exhibited increased expression of ER stress marker genes and augmented hepatic lipid accumulation after tunicamycin treatment. However, these changes were attenuated in inducible Fgf 21 transgenic mice. We also observed that Fgf21-null mice with leptin deficiency displayed increased hepatic ER stress response and liver injury, accompanied by deteriorated metabolic variables.

Conclusions/interpretation Our results suggest that FGF21 plays an important role in the adaptive response to ER stress- or obesity-induced hepatic metabolic stress.

Keywords ATF4 - eIF2 $\alpha \cdot$ ER stress $\cdot$ FGF2 $1 \cdot$ Insulin resistance $\cdot$ Lipid accumulation $\cdot$ Liver injury $\cdot$ Obesity

$\begin{array}{ll}\text { Abbreviations } \\ \text { ALT } & \text { Alanine aminotransferase } \\ \text { AMPK } & \text { AMP-activated protein kinase } \\ \text { AST } & \text { Aspartate aminotransferase } \\ \text { ATF4 } & \text { Activating transcription factor } 4 \\ \text { ATF6 } \alpha & \text { Activating transcription factor } 6 \alpha \\ \text { BAT } & \text { Brown adipose tissue } \\ \text { BIP } & \text { Binding immunoglobulin protein } \\ \text { CHOP } & \text { CCAAT/enhancer binding protein homologous } \\ & \text { protein }\end{array}$

Abbreviations

AMPK AMP-activated protein kinase

AST Aspartate aminotransferase

ATF4 Activating transcription factor 4

ATF $6 \alpha \quad$ Activating transcription factor $6 \alpha$

BAT Brown adipose tissue

BIP Binding immunoglobulin protein protein 


$\begin{array}{ll}\text { eIF2 } \alpha & \text { Eukaryotic translation factor } 2 \alpha \\ \text { ER } & \text { Endoplasmic reticulum } \\ \text { FGF21 } & \text { Fibroblast growth factor } 21 \\ \text { IRE1 } \alpha & \text { Inositol-requiring } 1 \alpha \\ \text { ITT } & \text { Insulin tolerance test } \\ \text { MEFs } & \text { Mouse embryonic fibroblasts } \\ \text { NAFLD } & \text { Nonalcoholic fatty liver disease } \\ \text { PERK } & \text { PKR-like endoplasmic reticulum kinase } \\ \text { PTT } & \text { Pyruvate tolerance test } \\ \text { SIRT1 } & \text { Sirtuin1 } \\ \text { TG } & \text { Triacylglycerol } \\ \text { UPR } & \text { Unfolded protein response } \\ \text { WAT } & \text { White adipose tissue } \\ \text { XBP1 } & \text { X-box binding protein 1 } \\ \text { XBP1s } & \text { Spliced X-box binding protein 1 }\end{array}$

\section{Introduction}

Fibroblast growth factor 21 (FGF21) is an endocrine hormone produced predominantly in the liver but also in white adipose tissue (WAT), brown adipose tissue (BAT), pancreas and skeletal muscle $[1,2]$. In starvation, FGF21 promotes lipolysis, beta oxidation or ketogenesis [3], indicating that FGF21 is a critical regulator of lipid homeostasis in adaptation to starvation. FGF21 can improve deteriorated metabolic variables in obese diabetic humans and in animal models of obesity and diabetes [4-7]. In addition, FGF21 plays a protective role in toxin-induced tissue injury $[8,9]$.

Protein folding occurs in the endoplasmic reticulum (ER). Perturbations of ER homeostasis cause accumulation of misfolded proteins in the ER lumen, triggering the unfolded protein response (UPR), an adaptive programme to resolve misfolded protein accumulation in the ER [10]. The UPR is regulated through three ER transmembrane sensors: inositol-requiring $1 \alpha$ (IRE1 $\alpha$ ), activating transcription factor $6 \alpha$ (ATF6 $\alpha$ ) and PKR-like ER kinase (PERK) [10]. X-box binding protein 1 (XBP1) and eukaryotic translation factor $2 \alpha$ (eIF $2 \alpha$ )-activating transcription factor 4 (ATF4) participate in various adaptive responses to ER stress downstream of IRE1 $\alpha$ and PERK, respectively. Unresolved ER stress leads to the development and progression of various diseases such as obesity and diabetes [11, 12].

Recent studies reported that FGF21 is increased in the liver in mouse models of and humans with obesity or nonalcoholic fatty liver disease (NAFLD) [13-15]. However, little is known about the functional role and molecular mechanism of FGF21 induction in these conditions. As ER stress is elevated in obesity [16], we investigated the relationship between ER stress and FGF21 induction. We found that FGF21 is induced by ER stress in a PERK-eIF2 $\alpha$-ATF4-dependent manner. In addition, we observed that FGF21 deletion accelerates ER stress-induced hepatic injury or lipid accumulation, and exacerbates obesity-induced ER stress and metabolic deterioration. These results suggest that FGF21 may play a role in the adaptive response to ER stress induced by a pharmacological ER stressor or obesity.

\section{Methods}

Animal experiments $\mathrm{Fg} 2 \mathrm{I}^{+/+}$and $\mathrm{Fg} f 2 \mathrm{1}^{-/-}$mice have been described elsewhere [17]. $F g f 21^{+/-}$mice were crossed with $o b / w$ mice (Jackson Laboratory, Bar Harbor, ME, USA) to generate $\mathrm{Fg} f 2 \mathrm{I}^{+/+} \mathrm{ob} / \mathrm{ob}$ and $\mathrm{Fg} f 2 \mathrm{I}^{-/-} \mathrm{ob} / \mathrm{ob}$ mice. Mice with a liver-specific defect in eIF $2 \alpha$ phosphorylation $\left(\right.$ Eif $2 \alpha^{\mathrm{A} / \mathrm{A}} / f T g /$ Alfp-Cre) were generated by breeding Eif $2 \alpha^{\mathrm{A} / \mathrm{A}} / f T g$ mice [18] with Eif $2 \alpha^{\mathrm{S} / \mathrm{A}} / A l f p$-Cre mice. Liver-specific inducible $\mathrm{Fg} f 21$ transgenic (Apoe-rtTA*M2/TetO-Fgf21) mice were generated using a tetracycline-inducible system. Fgf21-null and inducible $F g f 21$-transgenic mice were maintained in a specific pathogen-free facility of Samsung Biomedical Research Institute. Eif $2 \alpha$ mutant mice were maintained under a specific pathogen-free condition in the laboratory animal care facility of the University of Ulsan. All animal experiments were conducted in accordance with the guidelines of the Institutional Animal Care and Use Committee of Samsung Biomedical Research Institute or University of Ulsan. See Electronic Supplementary Material (ESM) Methods for details.

GTT, insulin tolerance test and pyruvate tolerance test GTTs and pyruvate tolerance tests (PTTs) were performed in overnight-fasted mice with intraperitoneal injection of glucose $(1 \mathrm{~g} / \mathrm{kg})$ and pyruvate $(1 \mathrm{~g} / \mathrm{kg})$, respectively. An insulin tolerance test (ITT) was conducted in $6 \mathrm{~h}$-fasted mice with an intraperitoneal injection of insulin $(1 \mathrm{U} / \mathrm{kg})$. Blood glucose levels were measured with an Accu-Check glucometer (Roche, Mannheim, Germany).

Ex vivo glucose-stimulated insulin secretion assay Pancreatic islets were isolated from female $\mathrm{Fg} f 2 \mathrm{I}^{+/+} \mathrm{ob} / \mathrm{ob}$ and Fgf $21^{-/-}$ob/ob mice by collagenase digestion and Biocoll (Biochrom AG, Berlin, Germany) gradient centrifugation. See ESM Methods for details.

Plasmid constructs and reagents See ESM Methods for details.

Generation of adenovirus Adenoviruses expressing ATF4 or Flag- $X B P 1 s$ were generated by homologous recombination between a linearised transfer vector (pAd-Track-ATF4 or 
pAd-Track-Flag-XBP1s) and an adenoviral backbone vector (pAd-Easy).

Cell culture FaO, AML12, HepG2 cells, primary mouse hepatocytes and mouse embryonic fibroblasts (MEFs) deficient in UPR genes were maintained at $37^{\circ} \mathrm{C}$ in a humid atmosphere of $5 \% \mathrm{CO}_{2}$. See ESM Methods for details.

Luciferase assay See ESM Methods for details.

siRNA transfection See ESM Methods for details.

RNA analysis Real-time RT-PCR was conducted using SYBR Green Master Mix (Takara, Otsu, Shiga, Japan) and genespecific primers (ESM Table 1) in ABI Prism 7000 (Applied Biosystems, Foster City, CA, USA). Relative expression values of specific genes were normalised to L32 mRNA. See ESM Methods for details.

Immunoblot analysis See ESM Methods for details.

Histology and staining analysis Liver and pancreas tissues were fixed with $10 \%$ neutral buffered formalin and $4 \%$ paraformaldehyde to make paraffin- and optimal cutting temperature-embedded blocks, respectively. See ESM Methods for details.

Blood chemistry and metabolite analysis See ESM Methods for details.

Statistical analysis All values are expressed as mean \pm SEM. Statistical significance was tested with the unpaired two-tailed Student's $t$ test using GraphPad Prism Version 5.02 Software (La Jolla, CA, USA). A $p$ value of less than 0.05 was considered significant.

\section{Results}

ER stress induces FGF21 expression through PERK-eIF2 $\alpha-$ ATF4 pathway in vitro To analyse the effect of ER stress on FGF21 expression in hepatocytes, mouse AML12 cells were treated with chemical ER stressors, tunicamycin and thapsigargin. We found that, remarkably, both tunicamycin and thapsigargin induced $F g f 21$ gene expression as well as upregulation of several UPR genes, including spliced $X b p 1$ (Xbp1s), Bip, Chop (also known as Ddit3) and Grp94 (Fig. 1a, b). Additionally, FGF21 expression was increased in rat $\mathrm{FaO}$, human $\mathrm{HepG} 2$ and primary mouse hepatocytes treated with ER stressors (Fig. 1b, c). In parallel, FGF21 level was augmented in culture supernatant fractions of $\mathrm{FaO}$ cells treated with ER stressors (Fig. 1d). These results demonstrate that ER stress induces FGF21 expression in various hepatocyte cell lines and primary hepatocytes.

We next investigated the mechanisms by which ER stress increases FGF21 expression using MEFs deficient in ER stress sensors. $F g f 21$ induction by ER stressors was much weaker in $\mathrm{Perk}^{-/-}$MEFs compared with control MEFs (Fig. 1e), while ER stressor-induced $F g f 21$ expression in

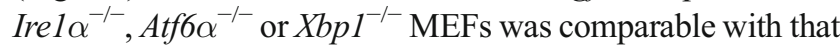
in control MEFs (ESM Fig. 1a-c). Furthermore, adenovirusmediated overexpression of XBP1s had no effect on FGF21 expression (ESM Fig. 1d). We next tested whether eIF $2 \alpha$ and ATF4 downstream of PERK induce FGF21 expression. As expected, two ER stressors increased $F g f 21$ mRNA levels in Eif $2 \alpha^{\mathrm{S} / \mathrm{S}}$ (wild-type) and $\mathrm{Atf} 4^{+/+} \mathrm{MEFs}$, while $\mathrm{Fg} 21$ induction was markedly attenuated in Eif2 $\alpha^{\mathrm{A} / \mathrm{A}}$ MEFs (harbouring S51A mutation) and Atf4 ${ }^{-/-}$MEFs (Fig. 1f, g). ATF4 knockdown consistently suppressed tunicamycin-induced $F g f 21$ expression (Fig. 1h, i), while ATF4 overexpression increased $F G F 21$ mRNA expression and $F g f 21$ promoter activity (Fig. 1j, k). However, XBP1s or ATF6 $\alpha$ overexpression had no effect on luciferase activity (Fig. 1k). Moreover, mutations of putative ATF4-response elements abolished the increase in Fgf21 promoter activity by ER stressors (Fig. 11), indicating that two ATF4-response elements are required for ER stressinduced FGF21 expression. Together, these findings suggest that the PERK-eIF2 $\alpha$-ATF4 pathway is critical for ER stressinduced FGF21 expression in vitro.

Hepatic eIF $2 \alpha-A T F 4$ pathway is required for ER stressinduced FGF21 expression in vivo We investigated whether or not ER stress stimulates FGF21 expression in vivo. When we injected tunicamycin into male C57BL/6 mice, hepatic $F g f 21$ and UPR gene expression was induced accompanied by elevated serum FGF21 levels (Fig. 2a-c, ESM Fig. 2). However, we did not observe a difference in $F g f 21$ expression in other tissues such as WAT or skeletal muscle (Fig. 2b), whereas $F g f 21$ expression in BAT appeared to be increased by tunicamycin administration (Fig. 2b). This finding indicates that hepatic FGF21 induction is largely responsible for elevated circulating FGF21 level after tunicamycin administration.

To study the importance of the eIF2 $\alpha$-ATF4 axis in FGF2 1 induction by ER stress in vivo, we generated liver-specific Eif $2 \alpha^{\mathrm{A} / \mathrm{A}}$ mice (Eif2 $\left.\alpha^{\mathrm{A} / \mathrm{A}} / f T g / A l f p-C r e\right)$ with a homozygous Ser51Ala mutation in Eif2 $\alpha$ alleles of the liver (Fig. 2d). Phosphorylation of eIF $2 \alpha$ in the liver was not observed in Eif $2 \alpha^{\mathrm{A} / \mathrm{A}} / f T g / A l f p$-Cre mice (Fig. 2e), confirming a defect in hepatic eIF $2 \alpha$ phosphorylation in these mice. Importantly, we observed that hepatic expression of FGF21 or ATF4 and serum FGF21 level were markedly decreased in liverspecific Eif $2 \alpha^{\mathrm{A} / \mathrm{A}}$ mice compared with control heterozygous Eif $2 \alpha^{\mathrm{S} / \mathrm{A}} / f T g$ mice under ER stress condition (Fig. 2e-g). These data indicate that the eIF $2 \alpha-\mathrm{ATF} 4$ pathway is critical for FGF21 induction in response to ER stress in vivo. 

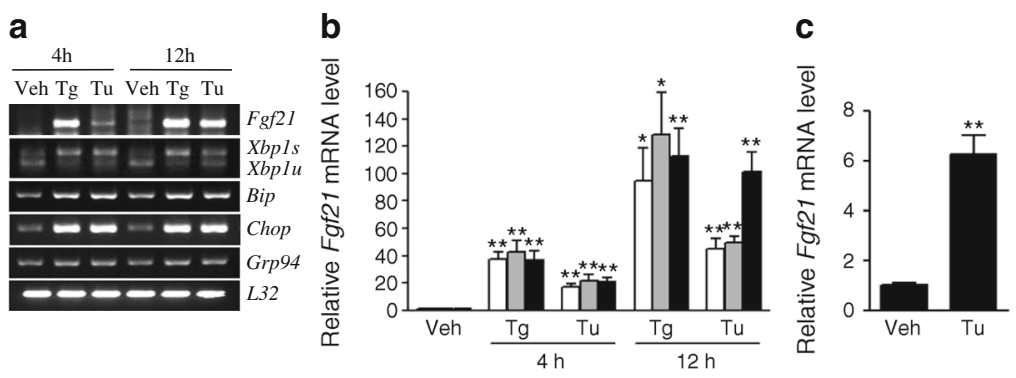

d
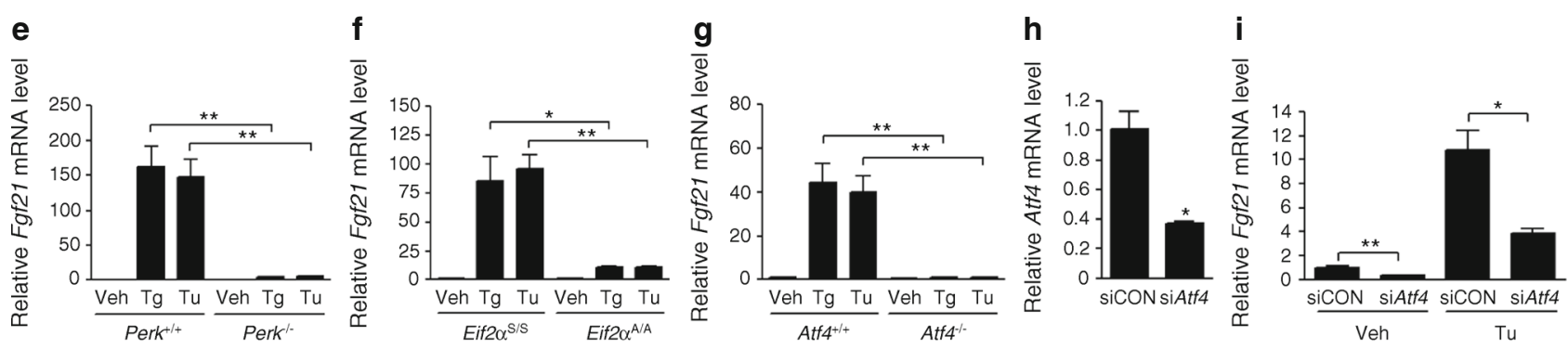
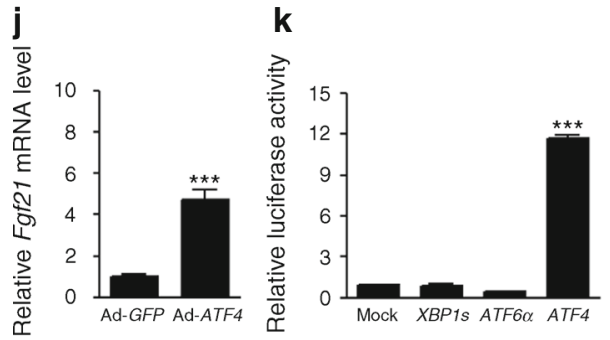

I

Fig. 1 The PERK-eIF2 $\alpha$-ATF4 pathway is indispensable for ER stressinduced FGF21 expression in vitro. (a) RT-PCR showing mRNA expression of $F g f 21$ and ER stress markers in AML12 cells treated with tunicamycin $(\mathrm{Tu}, 5 \mu \mathrm{g} / \mathrm{ml})$, thapsigargin $(\mathrm{Tg}, 1 \mu \mathrm{mol} / \mathrm{l})$ or dimethyl sulfoxide (DMSO, Veh) for indicated times $(n=3)$. (b) Relative Fgf 21 mRNA level in $\mathrm{FaO}$ (white bars), AML12 (grey bars) and HepG2 (black bars) cells treated with ER stressors $(n=3)$. Fgf 21 mRNA level normalised to L32 mRNA in Veh-treated cells is presented as $1 ;{ }^{*} p<0.05$, $* * p<0.01$ vs Veh. (c) Relative Fgf21 mRNA level in primary mouse hepatocytes treated with Tu for $4 \mathrm{~h}(n=3)$. Fgf $1 \mathrm{mRNA}$ level normalised to L32 mRNA in Veh-treated hepatocytes is presented as 1. (d) FGF21 level in culture supernatant fractions of $\mathrm{FaO}$ cells treated with ER stressors for $16 \mathrm{~h}(n=3) ; * * * p<0.001$ vs Veh. Relative $F g f 21$ mRNA level in (e) Perk $^{-/-}$, (f) Eif $2 \alpha^{-/-}$and (g) Atf4 ${ }^{-/-}$MEFs treated with ER

FGF21 suppresses ER stress response and alleviates ER stress-induced hepatic injury and lipid accumulation To study the physiological role of FGF21 induction by ER stress, we challenged $\mathrm{Fgfl} 2 \mathrm{I}^{-/-}$mice with tunicamycin and investigated changes in UPR signalling. As expected, there was no increase in serum FGF21 level in $F g f 21^{-1-}$ mice after tunicamycin administration (ESM Fig. 3a). Importantly, expression of UPR genes after tunicamycin treatment was significantly increased in the liver of $\mathrm{Fg} f 21^{-/-}$mice compared with control $F g f 21^{+/+}$mice (Fig. 3a). In parallel, levels of phosphorylated eIF2 $\alpha$, ATF4 and binding immunoglobulin protein (BIP) in the liver of $F g f 21^{-/-}$mice were higher compared with levels in control mice after tunicamycin treatment (Fig. 3b). Furthermore, induction of the pro-apoptotic
Mouse Fgf21 promoter-luciferase ATF4RE1 ATF4RE?
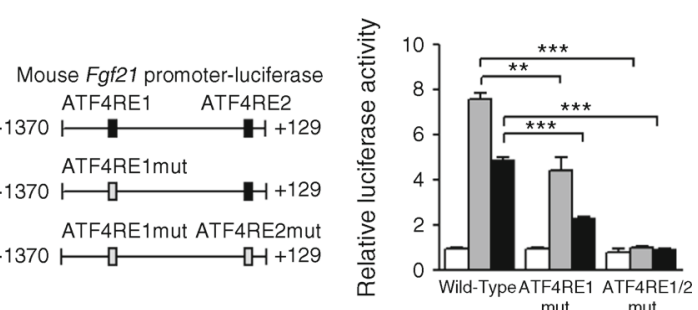

stressors for $12 \mathrm{~h}(n=3)$. $F g f 21$ mRNA level normalised to $L 32$ mRNA in Veh-treated wild-type MEFs is presented as 1. (h, i) Relative Atf4 or Fgf21 mRNA level in siCON- or siAtf4-transfected AML12 cells treated with Tu for $8 \mathrm{~h}(n=3)$. Atf4 and Fgf 21 mRNA level normalised to L32 mRNA in siCON-transfected cells and those treated with Veh are presented as 1, respectively. (j) Relative FGF21 mRNA level in HepG2 cells infected with adenovirus expressing GFP or $A T F 4$ for $36 \mathrm{~h}(n=3)$. $F G F 21$ mRNA level normalised to $L 32$ mRNA in Ad-GFP-infected cells is presented as 1. (k) Luciferase activity of $F g f 21$ promoter in $\mathrm{FaO}$ cells overexpressing XBP1s, ATF6 $\alpha$ or ATF4 for $24 \mathrm{~h}(n=3) ; * * * p<0.001 \mathrm{vs}$ Mock. (I) Luciferase activity of ATF4-response element (ATF4RE)-mutated FGF21 promoter in $\mathrm{FaO}$ cells treated with vehicle (white bars), $\mathrm{Tg}$ (grey bars) or Tu (black bars) for $12 \mathrm{~h}(n=3)$. Data are mean \pm SEM. ${ }^{*} p<0.05,{ }^{*} p<0.01, * * * p<0.001$

transcription factor CCAAT/enhancer binding protein homologous protein (CHOP) by tunicamycin was elevated in the liver of $\mathrm{Fg} 2 \mathrm{I}^{-/-}$mice compared with that of $\mathrm{Fg} 2 \mathrm{I}^{+/+}$mice (Fig. 3b), implying that FGF21 deletion may exacerbate liver injury caused by ER stress. In parallel, serum levels of alanine aminotransferase (ALT) and aspartate aminotransferase (AST) were increased in $\mathrm{Fgf}_{2} \mathrm{1}^{--}$mice under ER stress condition (Fig. 3c). These results suggest that FGF21 plays a protective role in ER stress-induced liver injury.

As ER stress can induce hepatic lipid accumulation [12], we next studied the physiological role of FGF21 in ER stressmediated hepatosteatosis. Importantly, $F g f 21^{-1-}$ mice exhibited elevated hepatic triacylglycerol (TG) accumulation compared with $F g f 21^{+/+}$mice after tunicamycin administration 
a

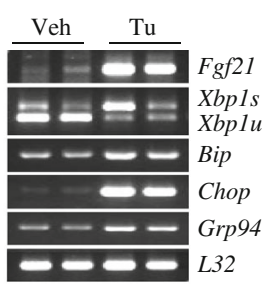

b

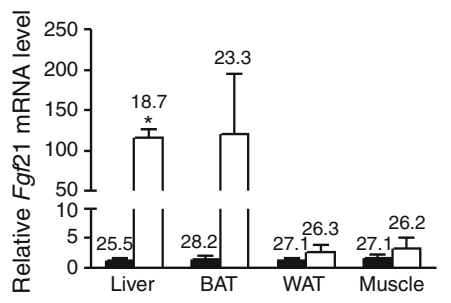

C

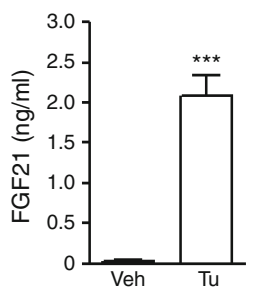

d

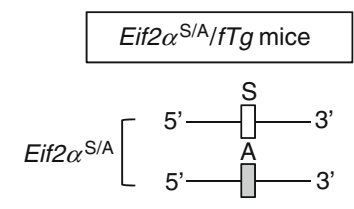

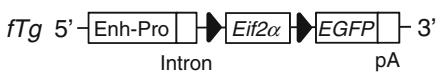

e

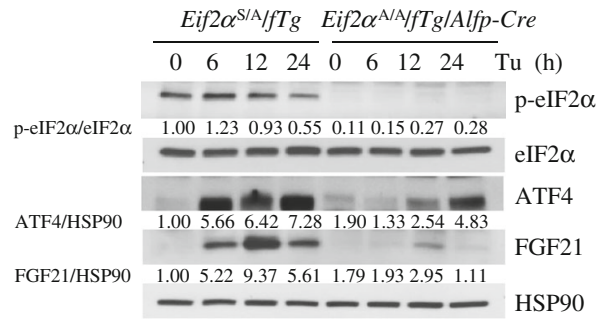

Fig. 2 Disruption of eIF2 $\alpha$-ATF4 pathway abrogates FGF21 induction in response to ER stress in vivo. (a) RT-PCR showing the mRNA expression of $F g f 21$ and ER stress markers in the liver of C57BL/6 mice after tunicamycin administration (Tu, $2 \mathrm{mg} / \mathrm{kg}$ ) or $2 \%$ DMSO (Veh) for 8 h. (b) Relative $F g f 21$ mRNA level $(n=4)$ and (c) serum FGF21 level $(n=6)$ in C57BL/6 mice after Veh (black bars) or Tu (white bars) administration for $24 \mathrm{~h}$. The number above the bars represents the mean $\mathrm{C}_{\mathrm{t}}$ value. $F g f 21 \mathrm{mRNA}$ level normalised to $L 32 \mathrm{mRNA}$ in Veh-treated tissue is presented as $1 ;{ }^{*} p<0.05$ vs Veh. (d) Schematic diagram for the generation of liver-specific Eif $2 \alpha^{\mathrm{A} / \mathrm{A}}$ mice. Eif $2 \alpha^{\mathrm{S} / \mathrm{A}} / f T g$ mice have a Ser51Ala mutation in one Eif $2 \alpha$ allele and LoxP sequences (black

(Fig. 3d, e). When we analysed changes in the expression of genes involved in lipid metabolism, the expression of fatty acid and TG synthesis genes such as Scd1, Adrp (also known as Plin2) and Dgat2 appeared to be increased in the liver of $\mathrm{Fg} 22 \mathrm{I}^{-/-}$mice compared with that of $\mathrm{Fg} f 2 \mathrm{I}^{+/+}$mice under ER stress condition (Fig. 3a). By contrast, we did not observe differences in the expression of genes such as Ppar, , Srebplc (also known as Srebfl) and Accl (Fig. 3a). The expression of $C d 36$, which is involved in lipid uptake, was also significantly increased in the liver of $F g f 21^{-/-}$mice compared with $\mathrm{Fgf} \mathrm{I}^{+/+}$mice (Fig. 3a). Additionally, we observed a tendency towards increased expression of the genes involved in beta oxidation, including Pparo and Mcad (also known as Acadm) in the liver of $F g f 21^{-/-}$mice compared with control mice after tunicamycin administration (ESM Fig. 3b), which is likely to be an adaptive response to excessive TG accumulation caused by FGF21 deletion. These results indicate that enhanced hepatic lipid synthesis and uptake may contribute to increased hepatic TG accumulation in $F g f 21^{-/-}$mice under ER stress condition.

We next investigated the effect of exogenous FGF21 on tunicamycin-induced ER stress and hepatic lipid accumulation using liver-specific inducible $F g f 21$-transgenic mice. As expected, inducible $F g f 21$-transgenic mice had increased serum FGF21 levels and elevated hepatic $F g f 21$ gene expression when fed chow diet containing doxycycline (Fig. 3f, ESM Fig. 4). Importantly, these mice showed attenuated expression of UPR genes and decreased hepatic lipid accumulation after tunicamycin administration (Fig. 3g, h). Together, these findings suggest that FGF21 expression is an adaptive response to ER stress to alleviate excessive liver injury and lipid accumulation. 
a

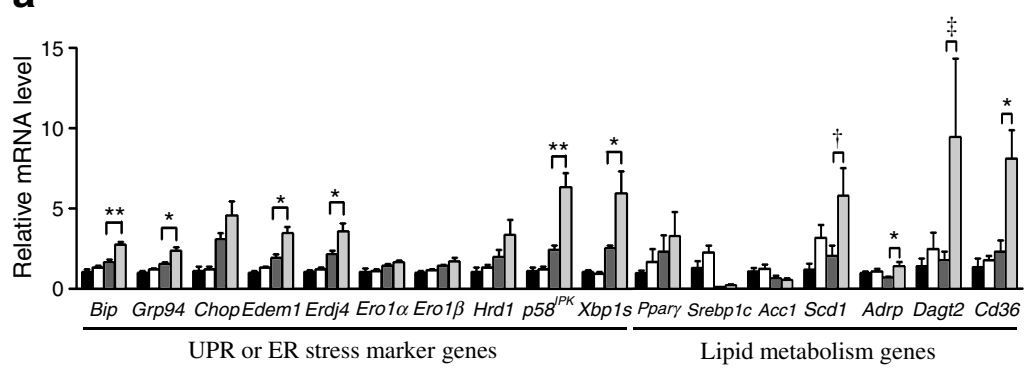

b

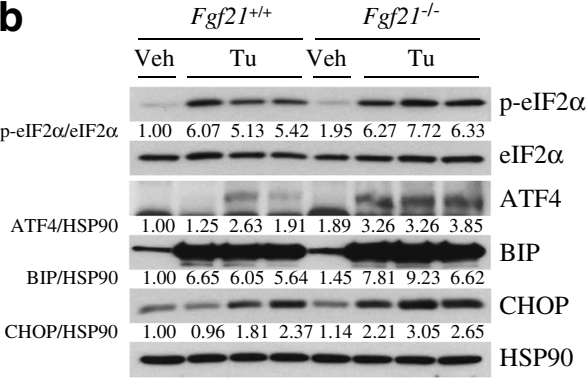

C

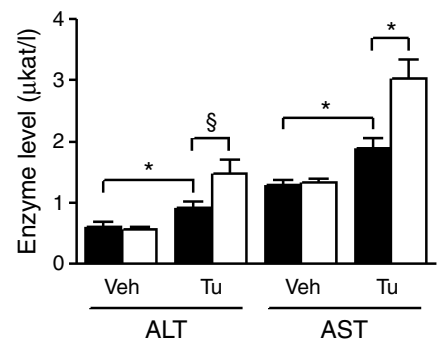

f

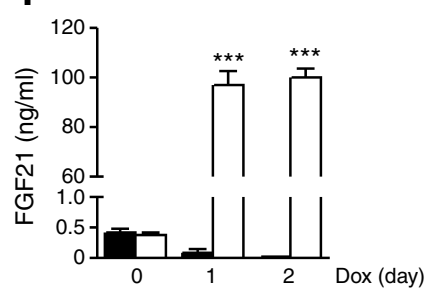

d

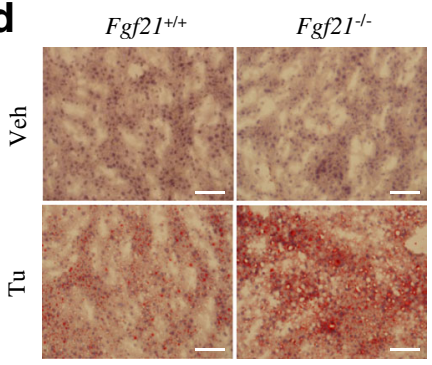

e

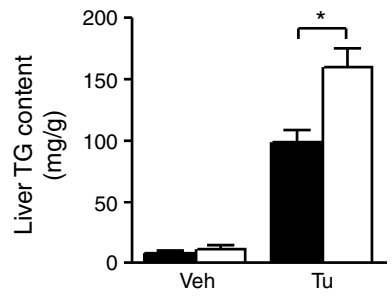

h
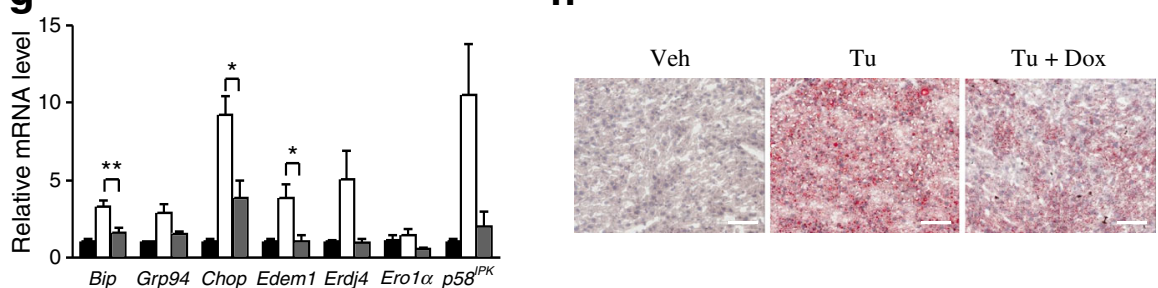

Bip Grp94 Chop Edem1 Erdj4 Ero1 $\alpha$ p58

Fig. 3 FGF21 alleviates upregulation of ER stress marker genes and attenuates liver injury or hepatic steatosis in response to ER stressors. (a) Relative mRNA level of genes of ER stress markers and lipid metabolism $(n=4-7)$, and (b) immunoblotting of ER stress markers in mice after administration of Vehicle (Veh) or tunicamycin (Tu, $1 \mathrm{mg} / \mathrm{kg}$ ) for $24 \mathrm{~h}$. mRNA levels normalised to L32 mRNA in the liver of Veh-treated $F g f 21^{+/+}$mice are presented as 1 . Black bars, $F g f 21^{+/+}+$Veh; white bars, $F g f 21^{-/-}+$Veh; dark grey bars, $F g f 21^{+/+}+\mathrm{Tu}$; light grey bars, $F g f 21^{-/}+$ Tu. (c) Serum ALT and AST levels $(n=5-11)$, (d) Oil Red O staining in the liver and (e) hepatic TG content $(n=5-11)$ in $F g f 21^{+/+}$(black bars) or $F g f 21^{-/-}$mice (white bars) after Tu administration $(1 \mathrm{mg} / \mathrm{kg}$ ) for $24 \mathrm{~h}$.

\section{Obesity-mediated FGF21 induction alleviates lipid-induced} ER stress, liver injury and metabolic deterioration Since ER stress occurs in the liver of obese mice [16], we investigated the pathophysiological role of FGF21 in obesity-induced hepatic ER stress using $\mathrm{Fgf} 2 \mathrm{1}^{-/-} \mathrm{ob} / \mathrm{ob}$ mice. Consistent with previous reports $[15,16], o b / o b$ mice exhibited increased hepatic $F g f 21$ expression and serum FGF21 levels compared with lean mice, together with upregulation of UPR genes and aggravated liver injury (Fig. 4a-c). Importantly, we found that both male and female $\mathrm{Fgf} 2 \mathrm{I}^{-/-} \mathrm{ob} / \mathrm{ob}$ mice displayed increased serum AST and ALT levels compared with their respective control $\mathrm{Fg} 22 \mathrm{I}^{+/+} \mathrm{ob} / \mathrm{ob}$ mice, although differences in male mice were marginal (Fig. 4d, e). In parallel, the expression of UPR genes was upregulated in the liver of female $F g f 21^{-1}$ - ob/ob mice compared with female controls (Fig. 4f). These results suggest that obesity-mediated FGF21 induction serves
Scale bars, $100 \mu \mathrm{m}$. (f) Serum FGF21 level in inducible Fgf21-transgenic (white bars) or control mice (black bars) with or without dietary doxycycline (Dox) for $1-2$ days $(n=3-11)$; ${ }^{* * *} p<0.001$ vs time-matched control mice. (g) Relative mRNA level of ER stress marker genes $(n=3)$ and (h) Oil Red $\mathrm{O}$ staining in the liver of inducible $F g f 21$-transgenic mice after Tu administration $(1 \mathrm{mg} / \mathrm{kg})$ for $24 \mathrm{~h}$. mRNA levels normalised to $L 32$ mRNA in the liver of Veh-treated mice are presented as 1. Black bars, Veh; white bars, Tu; grey bars, Tu+Dox. Scale bars, $100 \mu \mathrm{m}$. Data are mean \pm SEM. ${ }^{*} p<0.05,{ }^{*} p<0.01,{ }^{*} * * p<0.001,{ }^{\dagger} p=0.08,{ }^{\ddagger} p=0.17$, ${ }^{\S} p=0.09$

as a protective mechanism to ameliorate obesity-induced ER stress and liver injury.

Given the role of ER stress in obesity-related metabolic diseases [12], we next studied the role of FGF21 on obesitymediated metabolic derangement. Notably, both male and female $F g f 21^{-/}$ob/ob mice displayed increased random-fed or fasting glucose levels and worsened glucose tolerance/ insulin resistance compared with their respective control $F g f 21^{+/+} \mathrm{ob} / \mathrm{ob}$ mice without changes in body weight (Fig. 4g-j, ESM Fig. 5a-c), although the differences in fasting glucose level in female mice were marginal. However, we did not observe any difference in metabolic profile between nonobese $F g f 21^{+/+}$and $F g f 21^{-/-}$ mice of either sex (ESM Fig. 6), suggesting that FGF21 deletion exacerbates metabolic variables only in the metabolically stressed condition. 

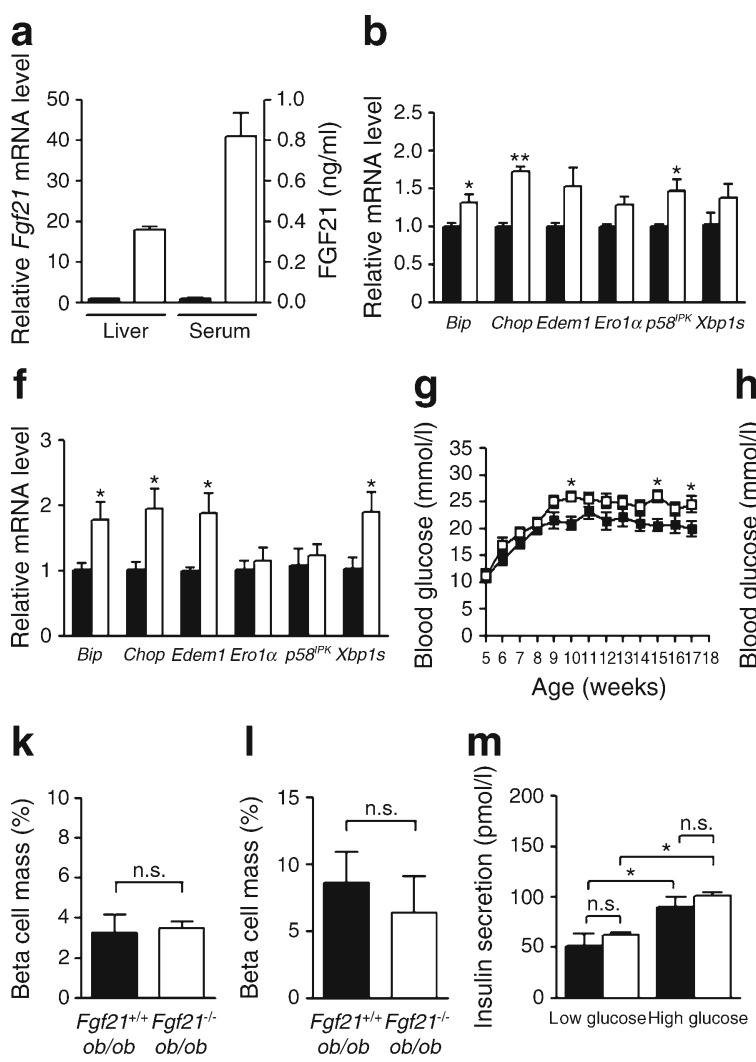

Fig. 4 FGF21 deletion aggravates obesity-induced ER stress and metabolic deterioration. (a) Hepatic Fgf21 mRNA level ( $n=3$ ), serum FGF21 level $(n=5)$, (b) relative mRNA level of hepatic ER stress marker genes $(n=3)$ and (c) serum ALT and AST levels $(n=5)$ in lean (black bars) and $o b / o b$ mice (white bars). mRNA levels normalised to L32 mRNA in the liver of lean mice are presented as 1. (d, e) Serum ALT and AST levels in $F g f 21^{+/+} o b / o b$ (black bars) and $F g f 21^{-/-} o b / o b$ mice (white bars) (d, male, $n=4$; e, female, $n=5$ ). (f) Relative hepatic mRNA level of ER stress marker genes in female mice $(n=4-5)$. mRNA levels normalised to $L 32$ mRNA in the liver of $F g f 21^{+/+} o b / o b$ mice are presented as 1. Black bars, $F g f 21^{+/+} \mathrm{ob} / \mathrm{ob}$; white bars, $\mathrm{Fgf} 2 \mathrm{I}^{-/-} \mathrm{ob} / \mathrm{ob}$. (g-l) Random-fed blood glucose level (g, male, $n=16-20 ; \mathbf{h}$, female, $n=18-21)$, GTT (i, male, $n=4 ; \mathbf{j}$, female, $n=5)$ and beta cell mass ( $\mathbf{k}$, male, $n=3 ; \mathbf{l}$, female, $n=3)$ in both

Despite deteriorated glucose tolerance and insulin resistance, serum insulin level, beta cell mass, beta cell apoptosis/proliferation and glucose-stimulated insulin secretion from islets were not different between $\mathrm{Fg} f 2 \mathrm{I}^{-/} \mathrm{ob} / \mathrm{ob}$ and $F g f 21^{+/+}$ob/ob mice (Fig. 4k-m, ESM Fig. 5d-f), suggesting that beta cell dysfunction is not involved in metabolic deterioration of $\mathrm{Fg} f 2 \mathrm{I}^{-/} \mathrm{ob} / \mathrm{ob}$ mice. As ER stress contributes to the increased gluconeogenesis in obesity and diabetes [19], we conducted a PTT in $\mathrm{Fg} 22 \mathrm{1}^{-/-} \mathrm{ob} / \mathrm{ob}$ mice. As expected, both male and female $\mathrm{Fg} f 2 \mathrm{I}^{-1-} \mathrm{ob} / \mathrm{ob}$ mice exhibited impaired pyruvate tolerance compared with $F g f 21^{+/+} o b / o b$ mice, accompanied by elevated expression of gluconeogenesis-related genes. However, differences in the PTTs of male mice were marginal (Fig. 4n-p), implying that deteriorated glucose tolerance is probably due to increased hepatic glucose production. Nonetheless, hepatic lipid content and serum levels of metabolites, such as TG, cholesterol and NEFA, were not

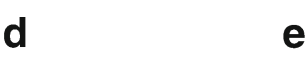

e
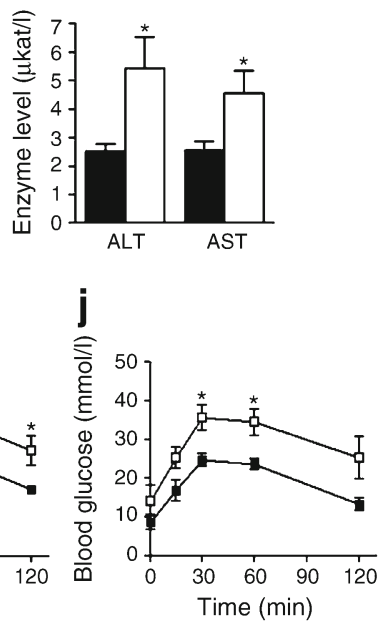

Age (weeks)
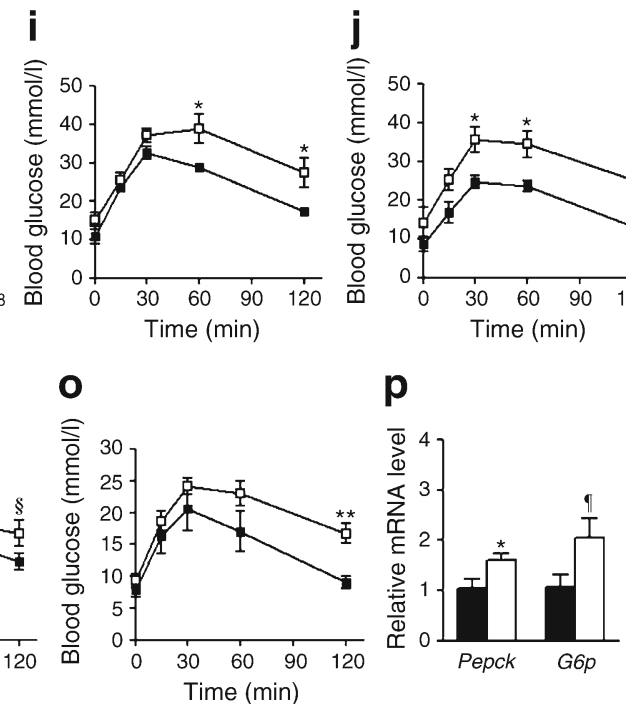

sexes; beta cell mass is measured as beta cell area/pancreas area. ${ }^{*} p<0.05$ vs age- or time-matched $F g f 21^{+/+} o b / o b$ mice. Black bars or squares, $\mathrm{Fgf} 2 \mathrm{I}^{+/+} \mathrm{ob} / \mathrm{ob}$; white bars or squares, $\mathrm{Fg} f 21^{-/-} \mathrm{ob} / \mathrm{ob}$. (m) Ex vivo glucose-stimulated insulin secretion from islets of female mice $(n=5)$. Black bars, $F g f 21^{+/+} o b / o b$; white bars, $F g f 21^{-/} o b / o b$. (n, o) PTT (n, male, $n=10-12$; o, female, $n=5-11)$. Black squares, $F g f 21^{+/+} o b / o b$; white squares, $F g f 21^{-/}$ob/ob. ${ }^{* *} p<0.01$ vs time-matched $F g f 21^{+/+} o b / o b$ mice. (p) Relative hepatic mRNA level of gluconeogenesis-related genes in female mice $(n=4-5)$. mRNA levels normalised to L32 mRNA in the liver of $\mathrm{Fg} 2 \mathrm{I}^{+/+} \mathrm{ob} / \mathrm{ob}$ mice are presented as 1 . Black bars, $\mathrm{Fg} f \mathrm{I}^{+/+} \mathrm{ob} /$ $o b$; white bars, $F g f 21^{-1-} o b / o b$. Data are mean \pm SEM. ${ }^{*} p<0.05$, ${ }^{* *} p<0.01,{ }^{* * *} p<0.001,{ }^{\dagger} p=0.12,{ }^{\ddagger} p=0.14,{ }^{\S} p=0.09,{ }^{\circledR} p=0.08$. n.s., not significant

different between the two groups in both sexes (ESM Fig. 7a-d). Together, these data suggest that obesitymediated FGF21 induction plays a protective role in obesityinduced ER stress and metabolic deterioration.

\section{Discussion}

Recent studies showed that FGF21 expression is increased in the liver of patients with and mouse models of obesity and NAFLD [13-15]. However, the molecular mechanism of FGF21 induction and its pathophysiological function in these conditions is unclear. Here, we demonstrated that a PERKeIF $2 \alpha-$ ATF 4 pathway is critical for ER stress-induced FGF2 1 expression in vitro and in vivo. Additionally, we showed a protective role of FGF21 in chemical- or obesity-induced hepatic ER stress. 
It has been reported that FGF21 is induced by various stresses, including glucose starvation, cold and autophagy deficiency, which may play an important role in adaptation to these stresses [3, 20,21]. Additionally, a recent paper has reported that FGF21 levels are increased in hepatocytes and in mouse liver after treatment with an ER stressor [22]. However, the physiological or pathological significance of ER stressmediated FGF21 induction is unknown. Furthermore, molecular mechanisms of in vitro FGF21 induction by ER stress are unclear, although the effects of ATF4 overexpression on $F G F 21$ mRNA expression and $F G F 21$ promoter activity have been evaluated $[22,23]$. Here, we demonstrated the importance of a PERK-eIF2 $\alpha$-ATF4 pathway in ER stress-induced $F g f 21$ expression in vivo and in vitro, and the physiological role of endogenous FGF21 induction caused by ER stress. In addition to ATF4, CHOP, the downstream target of ATF4, has been shown to be partially involved in ER stress-induced Fgf 21 expression in primary hepatocytes [23]. However, we did not observe differences in $F g f 21$ expression between $\mathrm{Chop}^{+/+}$and $\mathrm{Chop}^{-/-}$MEFs under ER stress conditions (ESM Fig. 8). During the course of the revision of our paper, the IRE $1 \alpha-\mathrm{XBP} 1$ pathway has been reported to mediate ER stress-induced FGF21 expression [24], which is in contrast to our findings showing no significant role of IRE $1 \alpha$ and XBP1 in FGF21 induction by ER stress. There is no clear explanation regarding these discrepancies, but they might be attributed to the differences in cell lines (hepatocytes vs MEFs) or experimental procedures.

$\mathrm{Fg} f 21^{-/-}$mice exhibited an aggravated ER stress response and hepatic cell death compared with $F g f 21^{+/+}$mice after tunicamycin administration, while inducible $F g f 21$-transgenic mice had an attenuated ER stress response, indicating that FGF21 plays a protective role against ER stress. However, we did not further address the mechanisms by which FGF21 alleviates ER stress or ER stress-induced liver injury in the present study. Several possible mechanisms may be proposed. First, downstream target proteins of FGF21 may participate in FGF21-mediated attenuation of ER stress. It has been reported that FGF21 activates the AMP-activated protein kinase (AMPK)-Sirtuin1 (SIRT1) pathway [25], and AMPK or SIRT1 has a protective role against ER stress $[26,27]$. We, therefore, hypothesise that AMPK or SIRT1 may mediate the effects of FGF21 on ER stress or hepatic injury due to ER stress. Another possibility is that reduced ER stress is a result of FGF21-induced suppression of lipid accumulation, since excessive lipid overloading can cause ER stress in the liver [28]. Given the protective role of FGF21 against oxidative stress in cardiac damage [29], reduced oxidative stress may be a mechanism by which FGF21 alleviates ER stress-induced hepatic injury. Intriguingly, FGF21 has been reported to exert metabolic effects via its actions in other target organs such as brain and adipose tissue [30-32]. In particular, increased adiponectin levels derived from adipose tissue contribute to the metabolic improvement elicited by FGF21 [31, 32]. However, we did not observe changes of circulating adiponectin levels or adipose tissue Adipoq mRNA level in tunicamycintreated mice (ESM Fig. 9a, b). In addition, serum adiponectin level in $F g f 21^{-1-}$ mice was not different compared with $\mathrm{Fg} 2 \mathrm{I}^{+/+}$mice after tunicamycin administration (ESM Fig. 9c). Thus, we could exclude the contribution of adiponectin in the adaptive effect of FGF21 in response to ER stress. However, we still cannot eliminate the possibility that other changes in non-hepatic tissues contribute to the amelioration of hepatic ER stress by FGF21. Further studies will be necessary to elucidate the mechanisms underlying the protective role of FGF21 against ER stress.

As ER stress contributes to obesity-associated metabolic disease [16] and FGF21 expression is increased in patients with and mouse models of obesity [13-15], we studied the pathophysiological function of FGF21 in ER stress and metabolic alterations caused by obesity using $o b$ mice. While the metabolic effects of FGF21 deletion on diet-induced obesity models were variable [33, 34], FGF21 deletion exacerbated hepatic ER stress and worsened glucose tolerance/insulin resistance in $o b$ mice. However, alterations of these metabolic variables were not associated with beta cell failure. Our findings are inconsistent with previous reports showing beneficial effects of FGF21 on beta cell function and impairment of FGF21 signalling in islets of obese mice $[35,36]$. This discrepancy is probably due to differences between exogenous FGF21 administration vs endogenous FGF21. Although the role of FGF21 in the regulation of gluconeogenesis is controversial [37], we observed elevated gluconeogenesis in $F g f 21^{-/-}$ob/ob mice, which is consistent with previous reports showing suppressive effects of FGF21 on gluconeogenesis in obese conditions [5]. Together, our results suggest that endogenous FGF21 is important in alleviating ER stress and metabolic deterioration due to obesity.

In conclusion, our results indicate that FGF21 is induced in response to ER stress through the eIF $2 \alpha-$ ATF 4 axis, which serves as a compensatory mechanism to attenuate ER stressinduced liver injury and hepatic lipid accumulation. We also showed that FGF21 induction plays a protective role in obesity-related ER stress and metabolic deterioration. Thus, our findings provide new insights into the role of FGF21 in ER stress response and suggest an innovative therapeutic strategy for the treatment of ER stress-associated diseases such as NAFLD, obesity and diabetes.

Acknowledgements We thank D. Ron (University of Cambridge, Cambridge, UK) for Atf4 and Perk MEFs, R. J. Kaufman (Sanford Burnham Medical Research Institute, La Jolla, CA, USA) for Irel $\alpha$, Atf $6 \alpha$, Chop, Eif $2 \alpha$ and Xbp1 MEFs and L. H. Glimcher (Weill Cornell Medical College, New York, NY, USA) for Xbpl MEFs. 
Funding This work was supported by the Basic Science Research Program through the National Research Foundation of Korea funded by the Ministry of Education (2013R1A6A3A04065825 to KHK and 2014R1A6A1030318 to SHB) and the Global Research Laboratory Grant of the National Research Foundation of Korea (K2100400000310A0500-00310 to M-SL). M-SL received the National Research Foundation of Korea grant funded by the Korea government (MSIP) (2014M3A9D8034459)

Duality of interest The authors declare that there is no duality of interest associated with this manuscript.

Contribution statement $\mathrm{KHK}$ and M-SL are the guarantors of the work. SHK, KHK and M-SL conceived and designed the experiments. SHK, KHK, H-KK, M-JK, SHB, MK and NI contributed to the acquisition of data. SHK, KHK and M-SL analysed and interpreted the data. SHK, KHK, SHB and M-SL wrote the manuscript. All authors have revised the manuscript critically for important intellectual content and approved the final version to be published.

\section{References}

1. Nishimura T, Nakatake Y, Konishi M, Itoh N (2000) Identification of a novel FGF, FGF-21, preferentially expressed in the liver. Biochim Biophys Acta 1492:203-206

2. Fon Tacer K, Bookout AL, Ding X et al (2010) Research resource: comprehensive expression atlas of the fibroblast growth factor system in adult mouse. Mol Endocrinol 24:2050-2064

3. Inagaki T, Dutchak P, Zhao G et al (2007) Endocrine regulation of the fasting response by PPARalpha-mediated induction of fibroblast growth factor 21. Cell Metab 5:415-425

4. Kharitonenkov A, Shiyanova TL, Koester A et al (2005) FGF-21 as a novel metabolic regulator. J Clin Invest 115:1627-1635

5. Xu J, Lloyd DJ, Hale C et al (2009) Fibroblast growth factor 21 reverses hepatic steatosis, increases energy expenditure, and improves insulin sensitivity in diet-induced obese mice. Diabetes 58 : 250-259

6. Gaich G, Chien JY, Fu H et al (2013) The effects of LY2405319, an FGF21 analog, in obese human subjects with type 2 diabetes. Cell Metab 18:333-340

7. Coskun T, Bina HA, Schneider MA et al (2008) Fibroblast growth factor 21 corrects obesity in mice. Endocrinology 149:6018-6027

8. Ye D, Wang Y, Li H, et al (2014) FGF21 protects against acetaminophen-induced hepatotoxicity by potentiating PGC-1alphamediated antioxidant capacity in mice. Hepatology 60:977-989

9. Cheng X, Vispute SG, Liu J, Cheng C, Kharitonenkov A, Klaassen CD (2014) Fibroblast growth factor (Fgf) 21 is a novel target gene of the aryl hydrocarbon receptor (AhR). Toxicol Appl Pharmacol 278: 65-71

10. Walter P, Ron D (2011) The unfolded protein response: from stress pathway to homeostatic regulation. Science 334:1081-1086

11. Hetz C, Chevet E, Harding HP (2013) Targeting the unfolded protein response in disease. Nat Rev Drug Discov 12:703-719

12. Hotamisligil GS (2010) Endoplasmic reticulum stress and the inflammatory basis of metabolic disease. Cell 140:900-917

13. Dushay J, Chui PC, Gopalakrishnan GS et al (2010) Increased fibroblast growth factor 21 in obesity and nonalcoholic fatty liver disease. Gastroenterology 139:456-463

14. Li H, Fang Q, Gao F et al (2010) Fibroblast growth factor 21 levels are increased in nonalcoholic fatty liver disease patients and are correlated with hepatic triglyceride. J Hepatol 53:934-940

15. Zhang X, Yeung DC, Karpisek M et al (2008) Serum FGF21 levels are increased in obesity and are independently associated with the metabolic syndrome in humans. Diabetes 57:1246-1253

16. Ozcan U, Cao Q, Yilmaz E et al (2004) Endoplasmic reticulum stress links obesity, insulin action, and type 2 diabetes. Science 306:457461

17. Hotta Y, Nakamura H, Konishi M et al (2009) Fibroblast growth factor 21 regulates lipolysis in white adipose tissue but is not required for ketogenesis and triglyceride clearance in liver. Endocrinology 150:4625-4633

18. Back SH, Scheuner D, Han J et al (2009) Translation attenuation through eIF2alpha phosphorylation prevents oxidative stress and maintains the differentiated state in beta cells. Cell Metab 10:13-26

19. Ozcan U, Yilmaz E, Ozcan L et al (2006) Chemical chaperones reduce ER stress and restore glucose homeostasis in a mouse model of type 2 diabetes. Science 313:1137-1140

20. Fisher FM, Kleiner S, Douris N et al (2012) FGF21 regulates PGC1 alpha and browning of white adipose tissues in adaptive thermogenesis. Genes Dev 26:271-281

21. Kim KH, Jeong YT, Oh H et al (2013) Autophagy deficiency leads to protection from obesity and insulin resistance by inducing Fgf21 as a mitokine. Nat Med 19:83-92

22. Schaap FG, Kremer AE, Lamers WH, Jansen PL, Gaemers IC (2013) Fibroblast growth factor 21 is induced by endoplasmic reticulum stress. Biochimie 95:692-699

23. Wan XS, Lu XH, Xiao YC et al (2014) ATF4- and CHOP-dependent induction of FGF21 through endoplasmic reticulum stress. Biomed Res Int 2014:807874

24. Jiang S, Yan C, Fang QC et al (2014) Fibroblast growth factor 21 is regulated by the IRE1 $\alpha$-XBP1 branch of the unfolded protein response and counteracts endoplasmic reticulum stress-induced hepatic steatosis. J Biol Chem 289:29751-29765

25. Chau MD, Gao J, Yang Q, Wu Z, Gromada J (2010) Fibroblast growth factor 21 regulates energy metabolism by activating the AMPK-SIRT1-PGC-1alpha pathway. Proc Natl Acad Sci U S A 107:12553-12558

26. Terai K, Hiramoto Y, Masaki M et al (2005) AMP-activated protein kinase protects cardiomyocytes against hypoxic injury through attenuation of endoplasmic reticulum stress. Mol Cell Biol 25:9554-9575

27. Li Y, Xu S, Giles A et al (2011) Hepatic overexpression of SIRT1 in mice attenuates endoplasmic reticulum stress and insulin resistance in the liver. FASEB J 25:1664-1679

28. Ota T, Gayet C, Ginsberg HN (2008) Inhibition of apolipoprotein B100 secretion by lipid-induced hepatic endoplasmic reticulum stress in rodents. J Clin Invest 118:316-332

29. Planavila A, Redondo I, Hondares E et al (2013) Fibroblast growth factor 21 protects against cardiac hypertrophy in mice. Nat Commun 4:2019

30. Bookout AL, de Groot MH, Owen BM et al (2013) FGF21 regulates metabolism and circadian behavior by acting on the nervous system. Nat Med 19:1147-1152

31. Holland WL, Adams AC, Brozinick JT et al (2013) An FGF21adiponectin-ceramide axis controls energy expenditure and insulin action in mice. Cell Metab 17:790-797

32. Lin Z, Tian H, Lam KS et al (2013) Adiponectin mediates the metabolic effects of FGF21 on glucose homeostasis and insulin sensitivity in mice. Cell Metab 17:779-789

33. Dutchak PA, Katafuchi T, Bookout AL et al (2012) Fibroblast growth factor-21 regulates PPARgamma activity and the antidiabetic actions of thiazolidinediones. Cell 148:556-567

34. Adams AC, Coskun T, Cheng CC, LS OF, Dubois SL, Kharitonenkov A (2013) Fibroblast growth factor 21 is not required for the antidiabetic actions of the thiazoladinediones. Mol Metab 2: 205-214

35. Wente W, Efanov AM, Brenner M et al (2006) Fibroblast growth factor-21 improves pancreatic beta-cell function and survival by 
activation of extracellular signal-regulated kinase $1 / 2$ and Akt signaling pathways. Diabetes 55:2470-2478

36. So WY, Cheng Q, Chen L et al (2013) High glucose represses beta-klotho expression and impairs fibroblast growth factor 21 action in mouse pancreatic islets: involvement of peroxisome proliferator-activated receptor gamma signaling. Diabetes 62: 3751-3759

37. Emanuelli B, Vienberg SG, Smyth G et al (2014) Interplay between FGF21 and insulin action in the liver regulates metabolism. J Clin Invest 124:515-527 